Publ. Mat. 60 (2016), 273-287

DOI: 10.5565/PUBLMAT_60216_01

\title{
ENTIRE SPACELIKE $H$-GRAPHS IN LORENTZIAN PRODUCT SPACES
}

\author{
Henrique F. de Lima and Eraldo A. Lima Jr.
}

\begin{abstract}
In this work we establish sufficient conditions to ensure that an entire spacelike graph immersed with constant mean curvature in a Lorentzian product space, whose Riemannian fiber has sectional curvature bounded from below, must be a trivial slice of the ambient space.
\end{abstract}

2010 Mathematics Subject Classification: Primary: 53C42; Secondary: 53B30, $53 \mathrm{C} 50$.

Key words: Lorentzian product spaces, spacelike hypersurfaces, mean curvature, entire spacelike $H$-graphs.

\section{Introduction and statements of the results}

The last few decades have seen a steadily growing interest in the study of the geometry of spacelike hypersurfaces immersed in a Lorentzian space. Apart from physical motivations, from the mathematical point of view this is mostly due to the fact that such hypersurfaces exhibit nice Bernstein-type properties, and one can truly say that the first remarkable results in this branch were the rigidity theorems of Calabi in [10] and Cheng and Yau in [11], who showed (the former for $n \leq 4$, and the latter for general $n$ ) that the only maximal (that is, with zero mean curvature) complete noncompact spacelike hypersurfaces of the Lorentz-Minkowski space $\mathbb{L}^{n+1}$ are the spacelike hyperplanes. However, in the case that the mean curvature is a positive constant, Treibergs [20] astonishingly showed that there are many entire solutions of the corresponding constant mean curvature equation in $\mathbb{L}^{n+1}$, which he was able to classify by their projective boundary values at infinity.

On the other hand, Xin [21] and Aiyama [1], working independently, characterized spacelike hyperplanes as the only complete constant mean curvature spacelike hypersurfaces in $\mathbb{L}^{n+1}$ whose Gauss mapping image is contained in a geodesic ball of the $n$-dimensional hyperbolic space. Later on, Aledo and Alías [6], among other results, showed that a complete constant mean curvature spacelike hypersurface which lies between 
two parallel spacelike hyperplanes of $\mathbb{L}^{n+1}$ must be, in fact, a spacelike hyperplane.

It is also natural to treat these same questions in a wide class of Lorentzian manifolds. When the ambient space is a Lorentzian product space, Salavessa [19] considered spacelike graphs in $-\mathbb{R} \times M^{n}$ and, under the assumption that the Cheeger constant of the fiber $M^{n}$ is zero and some conditions on the second fundamental form at infinity, she concluded that if the spacelike graph has parallel mean curvature then the graph must be maximal. When $M^{n}$ is the hyperbolic space $\mathbb{H}^{n}$, for any constant $c \in \mathbb{R}$ the author described an explicit foliation of $-\mathbb{R} \times \mathbb{H}^{n}$ by hypersurfaces with constant mean curvature $c$. Meanwhile, Albujer $[\mathbf{2}]$ obtained new explicit examples of complete and non-complete entire maximal spacelike graphs in $-\mathbb{R} \times \mathbb{H}^{2}$.

Afterwards, Albujer and Alías [3] established Calabi-Bernstein results for maximal spacelike surfaces immersed into a Lorentzian product space $-\mathbb{R} \times M^{2}$. In particular, when $M^{2}$ is a Riemannian surface with nonnegative Gaussian curvature, they proved that any complete maximal spacelike surface in $-\mathbb{R} \times M^{2}$ must be totally geodesic. Besides, assuming that the fiber $M^{2}$ is non-flat, the authors concluded that it must be a slice $\{t\} \times M^{2}$. In [14], Li and Salavessa generalized such results of $[\mathbf{3}]$ to higher dimension and codimension.

In [5], the first author jointly with Albujer and Camargo established uniqueness results concerning complete spacelike hypersurfaces with constant mean curvature immersed in $-\mathbb{R} \times \mathbb{H}^{n}$. Next, Albujer and Alías [4] obtained some parabolicity criteria for maximal surfaces immersed into a Lorentzian product space $-\mathbb{R} \times M^{2}$, where $M^{2}$ is supposed to have nonnegative Gaussian curvature. As an application of their main result, they deduced that every maximal graph over a starlike domain $\Omega \subset M^{2}$ is parabolic. This allowed them to give an alternative proof of the nonparametric version of the Calabi-Bernstein theorem for entire maximal graphs in such ambient space. Later, the first author jointly with Parente [13] obtained a lower estimate of the index of relative nullity of complete maximal spacelike hypersurfaces immersed in a so-called Robertson-Walker spacetime and, in particular, we also proved a sort of weak extension of the Calabi-Bernstein theorem in Lorentzian product spaces. More recently, the authors [12] applied some generalized maximum principles in order to establish uniqueness results concerning complete spacelike hypersurfaces with constant mean curvature in $-\mathbb{R} \times M^{n}$, extending the results of [5].

Motivated by these works described above, in this article we deal with entire spacelike graphs $\Sigma(u)=\left\{(u(x), x) ; x \in M^{n}\right\}$ with constant mean 
curvature in a Lorentzian product space $-\mathbb{R} \times M^{n}$, whose Riemannian fiber $M^{n}$ has sectional curvature bounded from below. According to the current literature, since the mean curvature $H$ of $\Sigma(u)$ is supposed to be constant, we call $\Sigma(u)$ an entire spacelike $H$-graph. In this setting, we obtain the following Calabi-Bernstein type result:

Theorem 1. Let $\bar{M}^{n+1}=-\mathbb{R} \times M^{n}$ be a Lorentzian product space, such that the sectional curvature $K_{M}$ of its Riemannian fiber $M^{n}$ satisfies $K_{M} \geq-\kappa$, for some positive constant $\kappa$. Let $\Sigma(u)$ be an entire spacelike $H$-graph over $M^{n}$, with $u$ bounded and $\mathrm{H}_{2}$ bounded from below. If

$$
|D u|_{M}^{2} \leq \frac{|A|^{2}}{\kappa(n-1)+|A|^{2}},
$$

then $u \equiv t_{0}$ for some $t_{0} \in \mathbb{R}$.

Here, $H_{2}=\frac{2}{n(n-1)} S_{2}$ is the mean value of the second elementary symmetric function $S_{2}$ on the eigenvalues of the shape operator $A$ of $\Sigma(u)$, $D u$ stands the gradient of the smooth function $u: M^{n} \rightarrow \mathbb{R}$ in $M^{n}$ and $|D u|_{M}$ its norm, both with respect to the metric of $M^{n}$.

In the context of Lorentzian product spaces, we note that our restriction on the sectional curvature $K_{M}$ of the fiber $M^{n}$ in Theorem 1 is a weaker restriction when compared with the so-called null (timelike) convergence condition, which means that the Ricci curvature of the ambient space is nonnegative on null or lightlike (timelike) directions (for a thorough discussion about such convergence conditions, see for example $[\mathbf{7}, \mathbf{8}, \mathbf{9}, \mathbf{1 5}])$. Furthermore, through the example described in Remark 3, we see that Theorem 1 is sharp in the sense that it does not hold when the function $u$ is unbounded.

The proof of Theorem 1 is given in Section 3. From Theorem 1 jointly with Theorem 3.3 of $[\mathbf{3}]$, it is not difficult to see that we also get the following result, where 1 -maximal means that $H_{2}$ vanishes identically on the graph:

Corollary 1. Let $\bar{M}^{n+1}=-\mathbb{R} \times M^{n}$ be a Lorentzian product space, such that the sectional curvature $K_{M}$ of its Riemannian fiber $M^{n}$ is nonnegative. If $\Sigma(u)$ is an entire 1-maximal spacelike $H$-graph over $M^{n}$ with $u$ bounded, then $\Sigma(u)$ is totally geodesic. In addition, if $n=2$ and $K_{M}(p)>0$ at some point $p \in M^{2}$, then $u \equiv t_{0}$ for some $t_{0} \in \mathbb{R}$.

We observe that, when the ambient space is the Lorentz-Minkowski space $\mathbb{L}^{n+1}$, Corollary 1 reads as follows:

Corollary 2. The only bounded entire 1-maximal spacelike $H$-graphs over a spacelike hyperplane of $\mathbb{L}^{n+1}$ are the spacelike hyperplanes. 


\section{Preliminaries}

In what follows, we deal with a spacelike hypersurface $\Sigma^{n}$ immersed into an $(n+1)$-dimensional Lorentzian product space $\bar{M}^{n+1}$ of the form $\mathbb{R} \times M^{n}$, where $M^{n}$ is an $n$-dimensional connected Riemannian manifold and $\bar{M}^{n+1}$ is endowed with the Lorentzian metric

$$
\langle,\rangle=-\pi_{\mathbb{R}}^{*}\left(d t^{2}\right)+\pi_{M}^{*}\left(\langle,\rangle_{M}\right),
$$

where $\pi_{\mathbb{R}}$ and $\pi_{M}$ denote the canonical projections from $\mathbb{R} \times M$ onto each factor, and $\langle,\rangle_{M}$ is the Riemannian metric on $M^{n}$.

For simplicity, we will just write $\bar{M}^{n+1}=-\mathbb{R} \times M^{n}$ and $\langle\rangle=$, $-d t^{2}+\langle,\rangle_{M}$. In this setting, for each fixed $t_{0} \in \mathbb{R}$, we say that $M_{t_{0}}^{n}=\left\{t_{0}\right\} \times M^{n}$ is a slice, which is a totally geodesic spacelike hypersurface of $\bar{M}^{n+1}$. We recall that a smooth immersion $\psi: \Sigma^{n} \rightarrow-\mathbb{R} \times M^{n}$ of an $n$-dimensional connected manifold $\Sigma^{n}$ is said to be a spacelike hypersurface if the induced metric via $\psi$ is a Riemannian metric on $\Sigma^{n}$, which, as usual, is also denoted for $\langle$,$\rangle .$

Since $\partial_{t}=\left(\partial / \partial_{t}\right)_{(t, x)},(t, x) \in-\mathbb{R} \times M^{n}$, is a unitary timelike vector field globally defined on the ambient spacetime, then there exists a unique timelike unitary normal vector field $N$ globally defined on the spacelike hypersurface $\Sigma^{n}$ which is in the same time-orientation as $\partial_{t}$. By using Cauchy-Schwarz inequality, we get $\left\langle N, \partial_{t}\right\rangle \leq-1$ on $\Sigma^{n}$. We will refer to that normal vector field $N$ as the future-pointing Gauss map of the spacelike hypersurface $\Sigma^{n}$.

Let $\bar{\nabla}$ and $\nabla$ denote the Levi-Civita connections in $-\mathbb{R} \times M^{n}$ and $\Sigma^{n}$, respectively. Then the Gauss and Weingarten formulas for the spacelike hypersurface $\psi: \Sigma^{n} \rightarrow-\mathbb{R} \times M^{n}$ are given by

$$
\bar{\nabla}_{X} Y=\nabla_{X} Y-\langle A X, Y\rangle N
$$

and

$$
A X=-\bar{\nabla}_{X} N
$$

for every tangent vector fields $X, Y \in \mathfrak{X}(\Sigma)$. Here $A: \mathfrak{X}(\Sigma) \rightarrow \mathfrak{X}(\Sigma)$ stands for the shape operator (or Weingarten endomorphism) of $\Sigma^{n}$ with respect to the future-pointing Gauss map $N$.

As in $[\mathbf{1 7}]$, the curvature tensor $R$ of the spacelike hypersurface $\Sigma^{n}$ is given by

$$
R(X, Y) Z=\nabla_{[X, Y]} Z-\left[\nabla_{X}, \nabla_{Y}\right] Z
$$

where [ ] denotes the Lie bracket and $X, Y, Z \in \mathfrak{X}(\Sigma)$. Another fact well known is that the curvature tensor $R$ of the spacelike hypersurface $\Sigma^{n}$ can be described in terms of the shape operator $A$ and the curvature 
tensor $\bar{R}$ of the ambient spacetime $-\mathbb{R} \times M^{n}$ by the so-called Gauss equation given by

$$
R(X, Y) Z=(\bar{R}(X, Y) Z)^{\top}-\langle A X, Z\rangle A Y+\langle A Y, Z\rangle A X,
$$

for every tangent vector fields $X, Y, Z \in \mathfrak{X}(\Sigma)$.

Now, we consider two particular functions naturally attached to a spacelike hypersurface $\Sigma^{n}$ immersed into a Lorentzian product space $-\mathbb{R} \times M^{n}$, namely, the (vertical) height function $h=\left.\left(\pi_{\mathbb{R}}\right)\right|_{\Sigma}$ and the support function $\left\langle N, \partial_{t}\right\rangle$, where we recall that $N$ denotes the futurepointing Gauss map of $\Sigma^{n}$ and $\partial_{t}$ is the coordinate vector field induced by the universal time on $-\mathbb{R} \times M^{n}$.

Let us denote by $\bar{\nabla}$ and $\nabla$ the gradients with respect to the metrics of $-\mathbb{R} \times M^{n}$ and $\Sigma^{n}$, respectively. Then, a simple computation shows that the gradient of $\pi_{\mathbb{R}}$ on $-\mathbb{R} \times M^{n}$ is given by

$$
\bar{\nabla} \pi_{\mathbb{R}}=-\left\langle\bar{\nabla} \pi_{\mathbb{R}}, \partial_{t}\right\rangle \partial_{t}=-\partial_{t},
$$

so that the gradient of $h$ on $\Sigma^{n}$ is

$$
\nabla h=\left(\bar{\nabla} \pi_{\mathbb{R}}\right)^{\top}=-\partial_{t}^{\top}=-\partial_{t}-\left\langle N, \partial_{t}\right\rangle N,
$$

where ()$^{\top}$ denotes the tangential component of a vector field in $\mathfrak{X}\left(\bar{M}^{n+1}\right)$ along $\Sigma^{n}$. Thus, we get

$$
|\nabla h|^{2}=\left\langle N, \partial_{t}\right\rangle^{2}-1
$$

where || denotes the norm of a vector field on $\Sigma^{n}$. Since $\partial_{t}$ is parallel on $-\mathbb{R} \times M^{n}$, we have that

$$
\bar{\nabla}_{X} \partial_{t}=0
$$

for every tangent vector field $X \in \mathfrak{X}(\Sigma)$. Writing $\partial_{t}=-\nabla h-\left\langle N, \partial_{t}\right\rangle N$ along the hypersurface $\Sigma^{n}$ and using formulas (2.1) and (2.2), from (2.4) and (2.6) we get that

$$
\nabla_{X} \nabla h=\left\langle N, \partial_{t}\right\rangle A X
$$

for every tangent vector field $X \in \mathfrak{X}(\Sigma)$. Therefore, from $(2.7)$ we obtain that the Laplacian on $\Sigma^{n}$ of its height function $h$ is given by

$$
\Delta h=-n H\left\langle N, \partial_{t}\right\rangle,
$$

where $H=-\frac{1}{n} \operatorname{tr}(A)$ denotes the mean curvature of $\Sigma^{n}$ with respect to its future-pointing Gauss mapping $N$.

Moreover, from (2.4) and (2.6) we also have that

$$
X\left(\left\langle N, \partial_{t}\right\rangle\right)=-\left\langle A(X), \partial_{t}\right\rangle=-\left\langle X, A\left(\partial_{t}^{\top}\right)\right\rangle=\langle X, A(\nabla h)\rangle,
$$


for all $X \in \mathfrak{X}(\Sigma)$. Thus,

$$
\nabla\left\langle N, \partial_{t}\right\rangle=-A\left(\partial_{t}^{\top}\right)=A(\nabla h) .
$$

Supposing that $\Sigma^{n}$ is a constant mean curvature spacelike hypersurface of $-\mathbb{R} \times M^{n}$, as a particular case of Corollary 8.2 in [7] we also obtain that the Laplacian on $\Sigma^{n}$ of its support function $\left\langle N, \partial_{t}\right\rangle$ is given by

$$
\Delta\left\langle N, \partial_{t}\right\rangle=\left(\operatorname{Ric}_{M}\left(N^{*}, N^{*}\right)+|A|^{2}\right)\left\langle N, \partial_{t}\right\rangle,
$$

where $\operatorname{Ric}_{M}$ is the Ricci curvature of the fiber $M^{n}, N^{*}=N+\left\langle N, \partial_{t}\right\rangle \partial_{t}$ is the projection of $N$ onto $M^{n}$ and $|A|$ stands for the Hilbert-Schmidt norm of the shape operator $A$ of $\Sigma^{n}$.

\section{Proof of Theorem 1}

Let $-\mathbb{R} \times M^{n}$ be a Lorentzian product space. We recall that an entire graph over the fiber $M^{n}$ is determined by a smooth function $u \in C^{\infty}(M)$ and it is given by

$$
\Sigma(u)=\left\{(u(x), x) ; x \in M^{n}\right\} \subset-\mathbb{R} \times M^{n} .
$$

The metric induced on $M^{n}$ from the Lorentzian metric on the ambient space via $\Sigma(u)$ is

$$
\langle,\rangle=-d u^{2}+\langle,\rangle_{M}
$$

Remark 1. It can be easily seen from (3.1) that an entire graph $\Sigma(u)$ is a spacelike hypersurface if, and only if, $|D u|_{M}^{2}<1$. Note that, when the fiber $M^{n}$ is simply connected, every complete spacelike hypersurface in $-\mathbb{R} \times M^{n}$ is an entire graph in such space (see, for instance, Lemma 3.1 of $[\mathbf{3}])$. However, according to the examples of non-complete entire maximal graphs in $-\mathbb{R} \times \mathbb{H}^{2}$ due to Albujer in Section 3 of [2], we see that an entire spacelike graph in a Lorentzian product space is not necessarily complete, in the sense that the induced Riemannian metric (3.1) is not necessarily complete.

If $\Sigma(u)$ is an entire graph over the fiber $M^{n}$, with a straightforward computation we verify that the vector field

$$
N=\frac{1}{\sqrt{1-|D u|_{M}^{2}}}\left(\partial_{t}+D u\right)
$$

defines the future-pointing Gauss map of $\Sigma(u)$. 
Let us study the shape operator $A$ of $\Sigma^{n}(u)$ with respect its orientation given by (3.2). For any $X \in \mathfrak{X}(\Sigma(u))$, since $X=X^{*}-\left\langle D u, X^{*}\right\rangle_{M} \partial_{t}$, we have that

$$
A X=-\bar{\nabla}_{X} N=\left\langle D u, X^{*}\right\rangle_{M} \bar{\nabla}_{\partial_{t}} N-\bar{\nabla}_{X^{*}} N .
$$

Consequently, from (3.2), (3.3), and with aid of Proposition 7.35 of [17], we verify that

$$
A X=-\frac{1}{\sqrt{1-|D u|_{M}^{2}}} D_{X^{*}} D u-\frac{\left\langle D_{X^{*}} D u, D u\right\rangle_{M}}{\left(1-|D u|_{M}^{2}\right)^{3 / 2}} D u,
$$

where $D$ denotes the Levi-Civita connection in $M^{n}$ with respect to the metric $\langle,\rangle_{M}$.

From (3.4) we obtain that the mean curvature of $\Sigma(u)$ is given by

$$
n H=\operatorname{Div}\left(\frac{D u}{\sqrt{1-|D u|_{M}^{2}}}\right),
$$

where Div stands for the divergence operator on $M^{n}$ with respect to the metric $\langle,\rangle_{M}$.

In order to prove Theorem 1, we will need two key lemmas. The first one gives a suitable lower estimate for the Ricci curvature of a spacelike hypersurface immersed in $-\mathbb{R} \times M^{n}$.

Lemma 1. Let $\Sigma^{n}$ be a spacelike hypersurface immersed in a Lorentzian product space $-\mathbb{R} \times M^{n}$, whose sectional curvature $K_{M}$ of its fiber $M^{n}$ verifies $K_{M} \geq-\kappa$ for some positive constant $\kappa$. Then, for all $X \in \mathfrak{X}(\Sigma)$, the Ricci curvature of $\Sigma^{n}$ satisfies the following inequality

$$
\operatorname{Ric}(X, X) \geq-\kappa(n-1)\left(1+|\nabla h|^{2}\right)|X|^{2}-\frac{n^{2} H^{2}}{4}|X|^{2} .
$$

Proof: let us consider $X \in \mathfrak{X}(\Sigma)$ and a local orthonormal frame $\left\{E_{1}, \ldots\right.$, $\left.E_{n}\right\}$ of $\mathfrak{X}(\Sigma)$. Then, it follows from Gauss equation (2.3) that

$$
\begin{aligned}
\operatorname{Ric}(X, X) & =\sum_{i=1}^{n}\left\langle\bar{R}\left(X, E_{i}\right) X, E_{i}\right\rangle+n H\langle A X, X\rangle+\langle A X, A X\rangle \\
& =\sum_{i=1}^{n}\left\langle\bar{R}\left(X, E_{i}\right) X, E_{i}\right\rangle-\frac{n^{2} H^{2}}{4}|X|^{2}+\left|A X+\frac{n H}{2} X\right|^{2} .
\end{aligned}
$$

Moreover, we have that

$$
\begin{aligned}
\left\langle\bar{R}\left(X, E_{i}\right) X, E_{i}\right\rangle & =\left\langle R\left(X^{*}, E_{i}^{*}\right) X^{*}, E_{i}^{*}\right\rangle_{M} \\
& =K_{M}\left(X^{*}, E_{i}^{*}\right)\left(\left\langle X^{*}, X^{*}\right\rangle_{M}\left\langle E_{i}^{*}, E_{i}^{*}\right\rangle_{M}-\left\langle X^{*}, E_{i}^{*}\right\rangle_{M}^{2}\right) .
\end{aligned}
$$


On the other hand, since $X^{*}=X+\left\langle X, \partial_{t}\right\rangle \partial_{t}, E_{i}^{*}=E_{i}+\left\langle E_{i}, \partial_{t}\right\rangle \partial_{t}$, and $\nabla h=-\partial_{t}^{\top}$, with a straightforward computation we see that

$$
\left\langle X^{*}, X^{*}\right\rangle_{M}\left\langle E_{i}^{*}, E_{i}^{*}\right\rangle_{M}=\left(1+\left\langle E_{i}, \nabla h\right\rangle^{2}\right)\left(|X|^{2}+\langle X, \nabla h\rangle^{2}\right)
$$

and

$$
\begin{aligned}
\left\langle X^{*}, E_{i}^{*}\right\rangle_{M}^{2}= & \left\langle X, E_{i}\right\rangle^{2}+2\langle X, \nabla h\rangle\left\langle E_{i}, \nabla h\right\rangle\left\langle X, E_{i}\right\rangle \\
& +\langle X, \nabla h\rangle^{2}\left\langle E_{i}, \nabla h\right\rangle^{2} .
\end{aligned}
$$

Therefore, since we are supposing that $K_{M} \geq-\kappa$ for some positive constant $\kappa$, we obtain

$$
\begin{aligned}
\sum_{i=1}^{n}\left\langle\bar{R}\left(X, E_{i}\right) X, E_{i}\right\rangle & \geq-\kappa\left((n-1)|X|^{2}+(n-2)\langle X, \nabla h\rangle^{2}+|X|^{2}|\nabla h|^{2}\right) \\
& \geq-\kappa(n-1)\left(1+|\nabla h|^{2}\right)|X|^{2},
\end{aligned}
$$

which jointly with (3.7) yields (3.6).

The second auxiliary lemma is the well known generalized maximum principle due to Omori [16] and Yau [22], which is quoted below.

Lemma 2. Let $\Sigma^{n}$ be an n-dimensional complete Riemannian manifold whose Ricci curvature is bounded from below and $\vartheta$ be a smooth function on $\Sigma^{n}$ which is bounded from below. Then, for each $\varepsilon>0$ there exists a point $p_{\varepsilon} \in \Sigma^{n}$ such that

$$
\inf _{\Sigma} \vartheta \leq \vartheta\left(p_{\varepsilon}\right)<\inf _{\Sigma} \vartheta+\varepsilon, \quad\left|\nabla \vartheta\left(p_{\varepsilon}\right)\right|<\varepsilon, \quad \text { and } \quad \Delta \vartheta\left(p_{\varepsilon}\right)>-\varepsilon .
$$

Now, we are in position to present the proof of Theorem 1.

Proof of Theorem 1: Observe first that, under the assumptions of the theorem, $\Sigma(u)$ is indeed a complete spacelike hypersurface. In fact, from (3.1) and the Cauchy-Schwarz inequality we get

$$
\langle X, X\rangle=\left\langle X^{*}, X^{*}\right\rangle_{M}-\left\langle D u, X^{*}\right\rangle_{M}^{2} \geq\left(1-|D u|_{M}^{2}\right)\left\langle X^{*}, X^{*}\right\rangle_{M},
$$

for every tangent vector field $X$ on $\Sigma(u)$.

On the other hand, we have that the Hilbert-Schmidt norm of the shape operator $A$ of $\Sigma(u)$ satisfies the following algebraic identity

$$
|A|^{2}=n^{2} H^{2}-n(n-1) H_{2} \text {. }
$$

Thus, since $H$ is constant and $H_{2}$ is supposed to be bounded from below, from (3.9) it holds that $\sup _{p \in \Sigma(u)}\left|A_{p}\right|^{2}<+\infty$. So, from (1.1) we see that there exists a constant $0<\alpha<1$ such that $|D u|_{M} \leq \alpha$. Hence, from (3.8) we get

$$
\langle X, X\rangle \geq\left(1-\alpha^{2}\right)\left\langle X^{*}, X^{*}\right\rangle_{M}
$$


This implies that $L \geq \sqrt{c} L_{M}$, where $L$ and $L_{M}$ denote the length of a curve on $\Sigma(u)$ with respect to the Riemannian metrics $\langle$,$\rangle and \langle,\rangle_{M}$, respectively, and $c=1-\alpha^{2}$. As a consequence, since we are supposing that $M^{n}$ is complete, then the induced metric on $\Sigma(u)$ from the metric of $-\mathbb{R} \times M^{n}$ is also complete.

Now, let us consider on $\Sigma(u)$ the functions $\eta=1-e^{-k u}$, with $k \in \mathbb{N}$, and $W=\sqrt{1-|D u|_{M}^{2}}$. Since we are supposing that $u$ is bounded, we have that the function $\vartheta=\eta W$ is bounded from below. On the other hand, since $H$ is constant and taking into account hypothesis (1.1) jointly with (3.12), from Lemma 1 we have that the Ricci curvature of $\Sigma(u)$ is also bounded from below. Hence, we can apply Lemma 2 to the function $\vartheta$, obtaining a sequence of points $\left\{p_{k, \varepsilon}\right\}$ in $\Sigma(u)$ such that, for each fixed $k>0$,

$$
|\nabla \vartheta|\left(p_{k, \varepsilon}\right) \leq \varepsilon, \quad \vartheta\left(p_{k, \varepsilon}\right) \leq \inf _{\Sigma(u)} \vartheta+\varepsilon, \quad \text { and } \quad \Delta \vartheta\left(p_{k, \varepsilon}\right) \geq-\varepsilon .
$$

Computing $\Delta \vartheta$ we obtain

$$
\Delta \vartheta=\Delta(\eta W)=W \Delta \eta+\eta \Delta W+2\langle\nabla W, \nabla \eta\rangle .
$$

Therefore, since $W \nabla \eta=\nabla \vartheta-\eta \nabla W$, from (3.10) we get

$$
\Delta \vartheta=W \Delta \eta+\eta\left(\Delta W-2 \frac{|\nabla W|^{2}}{W}\right)+\frac{2}{W}\langle\nabla W, \nabla \vartheta\rangle .
$$

On the other hand, since $N=-\left\langle N, \partial_{t}\right\rangle \partial_{t}+N^{*}$ where $N^{*}$ denotes the projection of $N$ onto the fiber $M^{n}$, from equation (2.4) it is not difficult to see that $N^{* \top}=-\left\langle N, \partial_{t}\right\rangle \nabla u$ and $|\nabla u|^{2}=\left\langle N^{*}, N^{*}\right\rangle_{M}$. Here, we are taking into account that the height function $h$ of $\Sigma(u)$ is nothing but the function $u$ regarded as a function on $\Sigma(u)$. Thus, from (3.2) we obtain that

$$
|\nabla u|^{2}=\frac{|D u|_{M}^{2}}{1-|D u|_{M}^{2}}=\frac{1-W^{2}}{W} .
$$

Consequently, from (2.5) and (3.12) we have that

$$
\left\langle N, \partial_{t}\right\rangle=-\frac{1}{W} .
$$

Hence, taking into account that

$$
\Delta\left(\frac{1}{W}\right)=-\frac{1}{W^{2}}\left(\Delta W-\frac{2|\nabla W|^{2}}{W}\right),
$$

we can use formula (2.10) to rewrite (3.11) as

$$
\Delta \vartheta=W \Delta \eta-\eta\left(\operatorname{Ric}_{M}\left(N^{*}, N^{*}\right)+|A|^{2}\right) W+\frac{2}{W}\langle\nabla W, \nabla \vartheta\rangle .
$$


Hence, along the minimizing sequence $\left\{p_{k, \varepsilon}\right\}$, from (3.14) we get

$$
-\varepsilon \leq W \Delta \eta-\eta\left(\operatorname{Ric}_{M}\left(N^{*}, N^{*}\right)+|A|^{2}\right) W+\frac{2}{W}\langle\nabla W, \nabla \vartheta\rangle .
$$

So, using Cauchy-Schwartz inequality in (3.15), we obtain that

$$
-\varepsilon \leq W \Delta \eta-\eta\left(\operatorname{Ric}_{M}\left(N^{*}, N^{*}\right)+|A|^{2}\right) W+\frac{2 \varepsilon|\nabla W|}{W} .
$$

On the other hand, since we are assuming that $K_{M} \geq-\kappa$ for some positive constant $\kappa$, we have

$$
\operatorname{Ric}_{M}\left(N^{*}, N^{*}\right) \geq-\kappa(n-1)\left|N^{*}\right|_{M}^{2}=-\kappa(n-1)|\nabla u|^{2} .
$$

But, from (1.1) and (3.12) it holds that

$$
|\nabla u|^{2} \leq \frac{|A|^{2}}{\kappa(n-1)}
$$

Consequently, from (3.17) we obtain

$$
\operatorname{Ric}_{M}\left(N^{*}, N^{*}\right)+|A|^{2} \geq-\kappa(n-1)|\nabla u|^{2}+|A|^{2} \geq 0 .
$$

Furthermore, up to translation, we can assume $u>0$ and, hence, we have that $\eta>0$ on $\Sigma(u)$. Therefore, from (3.16) and (3.18) we get

$$
-\varepsilon\left(\frac{W+2|\nabla W|}{W^{2}}\right) \leq \Delta \eta
$$

Using the general formula $\Delta f(u)=f^{\prime} \Delta u+f^{\prime \prime}|\nabla u|$, we also have that

$$
\Delta \eta=e^{-k u}\left(k \Delta u-k^{2}|\nabla u|^{2}\right) .
$$

Thus, from (3.19) and (3.20) we obtain

$$
-\varepsilon\left(\frac{W+2|\nabla W|}{W^{2}}\right) \leq e^{-k u}\left(k \Delta u-k^{2}|\nabla u|^{2}\right) .
$$

Hence, taking into account (2.8) and (3.5), from (3.21) we must have

$$
-\varepsilon e^{k u}(W+2|\nabla W|) \leq\left(-n H k W-k^{2}|D u|_{M}^{2}\right) .
$$

We claim that $\nabla W$ is also bounded. Indeed, from (3.13) we have that

$$
\nabla W=-\frac{1}{\left\langle N, \partial_{t}\right\rangle^{2}} \nabla\left\langle N, \partial_{t}\right\rangle
$$

Hence, from (2.9) and (3.23) we get

$$
|\nabla W| \leq W^{2}|A||\nabla u| \leq W^{2} \frac{|A|^{2}}{\sqrt{\kappa(n-1)}} .
$$


Thus, letting $\varepsilon \rightarrow 0$ in (3.22) and taking the $\lim \sup$ on $\varepsilon$, we obtain the following estimate

$$
0 \leq-n|H| \limsup _{\varepsilon \rightarrow 0} W-k \limsup _{\varepsilon \rightarrow 0}|D u|_{M}^{2} .
$$

Now, multiplying (3.24) by $\frac{1}{k}$ and making $k \rightarrow \infty$ as we take the limsup over $k$ we get the next

$$
\limsup _{k \rightarrow \infty} \limsup _{\varepsilon \rightarrow 0}|D u|_{M}^{2}=0 .
$$

Consequently, since $W^{2}=1-|D u|_{M}^{2}$, we have

$$
\liminf _{k \rightarrow \infty} \liminf _{\varepsilon \rightarrow 0} W^{2}=1 .
$$

Since these sequences are minimizing, by Lemma 2 on an arbitrary point we have the ensuing

$$
\eta^{2} W^{2}\left(p_{k, \varepsilon}\right) \leq \eta^{2} W^{2}+\varepsilon
$$

which implies that

$$
\begin{aligned}
|D u|_{M}^{2} & \leq 1-\frac{\eta_{*}^{2}}{\eta^{2}} W^{2}\left(p_{k, \varepsilon}\right)+\frac{\varepsilon}{\eta^{2}} \\
& \leq 1-\left(1-e^{-k u_{*}}\right)^{2} W^{2}\left(p_{k, \varepsilon}\right)+\frac{\varepsilon}{\left(1-e^{-k u_{*}}\right)^{2}},
\end{aligned}
$$

where $\eta_{*}=\inf _{\Sigma(u)} \eta$ and $u_{*}=\inf _{\Sigma(u)} u$. Without loss of generality, denoting $u^{*}=\sup _{\Sigma(u)} u$, we can suppose that $u^{*} \geq u \geq u_{*}>0$. Thus,

$$
|D u|_{M}^{2} \leq 1-\left(1-e^{-k u_{*}}\right)^{2} W^{2}\left(p_{k, \varepsilon}\right)+\frac{\varepsilon}{\left(1-e^{-k u^{*}}\right)^{2}} .
$$

Since $\varepsilon$ does not appear in the left hand side of (3.27), we can take $\lim \sup _{\varepsilon \rightarrow 0}$ on both sides of (3.27) obtaining

$$
|D u|_{M}^{2} \leq 1-\left(1-e^{-k u_{*}}\right)^{2} \liminf _{\varepsilon \rightarrow 0} W^{2}\left(p_{k, \varepsilon}\right) .
$$

In an analogous way, taking $\lim \sup _{k \rightarrow \infty}$ on (3.28), we finally conclude that $|D u|_{M}^{2}=0$ on $\Sigma(u)$, that is, $u \equiv t_{0}$ for some $t_{0} \in \mathbb{R}$.

Remark 2. We recall that the Cheeger constant $\mathfrak{b}(M)$ of a complete Riemannian manifold $M^{n}$ is given by

$$
\mathfrak{b}(M)=\inf _{D} \frac{A(\partial D)}{V(D)}
$$

where $D$ ranges over all open submanifolds of $M^{n}$ with compact closure in $M^{n}$ and smooth boundary, and where $V(D), A(\partial D)$ are the volume of $D$ resp. the area of $\partial D$, relative to the metric of $M^{n}$. 
Returning to the context of Theorem 1, assuming that there exists an entire spacelike $H$-graph with $H>0$ and such that (1.1) holds, from (3.5) we can apply an argument due to Salavessa [18] to get

$$
\begin{aligned}
n H V(D) & \leq \int_{D} n H d V=\int_{D} \operatorname{Div}\left(\frac{D u}{\sqrt{1-|D u|_{M}^{2}}}\right) d V \\
& =\oint_{\partial D}\left\langle\frac{D u}{\sqrt{1-|D u|_{M}^{2}}}, \nu\right\rangle d A \leq \sqrt{\frac{n}{(n-1) \kappa}} H A(\partial D),
\end{aligned}
$$

where $\nu$ is the outward unit normal of $\partial D$. Yielding the following lower estimate for the Cheeger constant of the fiber $M^{n}$

$$
\sqrt{n(n-1) \kappa} \leq \mathfrak{b}(M) .
$$

Furthermore, recalling the stability operator $\mathcal{J}=\Delta+\operatorname{Ric}(N, N)+$ $|A|^{2}$, a spacelike $H$-hypersurface $\Sigma^{n}$ is said to be stable if

$$
\int_{\Sigma} \mathcal{J} f \cdot f \geq 0, \quad \forall f \in C_{0}^{2}(\Sigma) .
$$

We also note that, under the stated hypothesis of Theorem 1, entire spacelike $H$-graph is, in fact, a slice and therefore $\operatorname{Ric}\left(\partial_{t}, \partial_{t}\right)=0$ and $|A|^{2} \equiv 0$. Hence, in this case, from (3.29) we see that such graph is stable.

Remark 3. According to Example 4.4 of [12], taking $0<|a|<1$, we have that the entire vertical graph

$$
\Sigma(u)=\{(a \ln y, x, y) ; y>0\} \subset-\mathbb{R} \times \mathbb{H}^{2}
$$

is such that

$$
|D u|_{\mathbb{H}^{2}}^{2}=|a|^{2}
$$

and, hence, $\Sigma(u)$ is a complete spacelike surface in $-\mathbb{R} \times \mathbb{H}^{2}$. Moreover, with a straightforward computation we verify that $\Sigma^{2}(u)$ has constant mean curvature $H=-\frac{a}{2 \sqrt{1-a^{2}}}, H_{2}=0$, and

$$
|D u|_{\mathbb{H}^{2}}^{2}=\frac{|A|^{2}}{1+|A|^{2}} .
$$

Hence, we conclude that Theorem 1 does not hold when the function $u$ is unbounded.

Furthermore, since $\left\langle N, \partial_{t}\right\rangle$ is constant on $\Sigma(u)$, from formula (2.10) and taking into account equations (3.12) and (3.30), we get

$$
\Delta\left\langle N, \partial_{t}\right\rangle=\left(|A|^{2}-|\nabla h|^{2}\right)\left\langle N, \partial_{t}\right\rangle=0 .
$$


Consequently, according to the stability criteria given in (3.29), from equation (3.31) we also conclude that $\Sigma(u)$ constitutes a nontrivial example of stable surface in $-\mathbb{R} \times \mathbb{H}^{2}$. Therefore, concerning the context of Theorem 1 , we see that the stability of the entire spacelike $H$-graph cannot alone guarantee the uniqueness result.

\section{Acknowledgements}

The first author is partially supported by CNPq, Brazil, grant 300769/ 2012-1. The second author is partially supported by CAPES, Brazil. The authors would like to thank the referee for giving several valuable suggestions which improved the paper.

\section{References}

[1] R. Aiyama, On the Gauss map of complete space-like hypersurfaces of constant mean curvature in Minkowski space, Tsukuba J. Math. 16(2) (1992), 353-361.

[2] A. L. Albujer, New examples of entire maximal graphs in $\mathbb{H}^{2} \times \mathbb{R}_{1}$, Differential Geom. Appl. 26(4) (2008), 456-462. DOI: 10.1016/j.difgeo.2007.11.035.

[3] A. L. Albujer and L. J. Alías, Calabi-Bernstein results for maximal surfaces in Lorentzian product spaces, J. Geom. Phys. 59(5) (2009), 620-631. DOI : 10.1016/j.geomphys.2009.01.008.

[4] A. L. Albujer and L. J. Alías, Parabolicity of maximal surfaces in Lorentzian product spaces, Math. Z. 267(1-2) (2011), 453-464. DOI : $10.1007 / \mathrm{s} 00209-009-0630-8$.

[5] A. L. Albujer, F. E. C. Camargo, and H. F. De Lima, Complete spacelike hypersurfaces with constant mean curvature in $-\mathbb{R} \times \mathbb{H}^{n}$, J. Math. Anal. Appl. 368(2) (2010), 650-657. DOI: 10.1016/j.jmaa.2010.02.039.

[6] J. A. Aledo And L. J. Alías, On the curvatures of bounded complete spacelike hypersurfaces in the Lorentz-Minkowski space, Manuscripta Math. 101(3) (2000), 401-413. DOI: 10.1007/ s002290050223.

[7] L. J. Alías and A. G. Colares, Uniqueness of spacelike hypersurfaces with constant higher order mean curvature in generalized Robertson-Walker spacetimes, Math. Proc. Cambridge Philos. Soc. 143(3) (2007), 703-729. DOI: 10.1017/S0305004107000576. 
[8] L. J. Alías, A. Romero, And M. SÁnchez, Uniqueness of complete spacelike hypersurfaces of constant mean curvature in generalized Robertson-Walker spacetimes, Gen. Relativity Gravitation 27(1) (1995), 71-84. DOI: 10.1007/BF02105675.

[9] L. J. Alías, A. Romero, And M. SÁnchez, Spacelike hypersurfaces of constant mean curvature and Calabi-Bernstein type problems, Tohoku Math. J. (2) 49(3) (1997), 337-345. DOI: 10.2748/ $\mathrm{tmj} / 1178225107$.

[10] E. CAlabi, Examples of Bernstein problems for some nonlinear equations, in: "Global Analysis", Proc. Sympos. Pure Math. XV, Amer. Math. Soc., Providence, R.I., 1970, pp. 223-230.

[11] S.-Y. Cheng And S.-T. YAU, Maximal space-like hypersurfaces in the Lorentz-Minkowski spaces, Ann. of Math. (2) 104(3) (1976), 407-419. DOI : $10.2307 / 1970963$.

[12] H. F. DE Lima And E. A. Lima, JR., Generalized maximum principles and the unicity of complete spacelike hypersurfaces immersed in a Lorentzian product space, Beitr. Algebra Geom. 55(1) (2014), 59-75. DOI : $10.1007 / \mathrm{s} 13366-013-0137-7$.

[13] H. F. de Lima and U. L. Parente, On the geometry of maximal spacelike hypersurfaces immersed in a generalized RobertsonWalker spacetime, Ann. Mat. Pura Appl. (4) 192(4) (2013), 649-663. DOI : 10.1007/s10231-011-0241-y.

[14] G. Li And I. Salavessa, Graphic Bernstein results in curved pseudo-Riemannian manifolds, J. Geom. Phys. 59(9) (2009), 1306-1313. DOI : 10.1016/j.geomphys.2009.06.011.

[15] S. Montiel, Uniqueness of spacelike hypersurfaces of constant mean curvature in foliated spacetimes, Math. Ann. 314(3) (1999), 529-553. DOI : $10.1007 / \mathrm{s} 002080050306$.

[16] H. OMORI, Isometric immersions of Riemannian manifolds, $J$. Math. Soc. Japan 19(2) (1967), 205-214. DOI: 10.2969/jmsj/ 01920205.

[17] B. O'NeILL, "Semi-Riemannian geometry. With applications to relativity", Pure and Applied Mathematics 103, Academic Press, Inc. [Harcourt Brace Jovanovich, Publishers], New York, 1983.

[18] I. M. C. SAlavessa, Graphs with parallel mean curvature, Proc. Amer. Math. Soc. 107(2) (1989), 449-458. DOI: 10.2307/ 2047835.

[19] I. M. C. Salavessa, Spacelike graphs with parallel mean curvature, Bull. Belg. Math. Soc. Simon Stevin 15(1) (2008), 65-76. 
[20] A. E. Treibergs, Entire spacelike hypersurfaces of constant mean curvature in Minkowski space, Invent. Math. 66(1) (1982), 39-56. DOI : $10.1007 / \mathrm{BF} 01404755$.

[21] Y. L. XIN, On the Gauss image of a spacelike hypersurface with constant mean curvature in Minkowski space, Comment. Math. Helv. 66(4) (1991), 590-598.

[22] S.-T. YAU, Harmonic functions on complete Riemannian manifolds, Comm. Pure Appl. Math. 28 (1975), 201-228. DOI: 10.1002/cpa. 3160280203 .

Henrique F. de Lima:

Departamento de Matemática

Universidade Federal de Campina Grande

58429-970 Campina Grande

Paraíba, Brazil

E-mail address: henrique@dme.ufcg.edu.br

Eraldo A. Lima Jr.:

Departamento de Matemática

Universidade Federal do Ceará

60356-000 Fortaleza

Ceará, Brazil

E-mail address: eraldojrps21@gmail.com

Primera versió rebuda el 12 de juny de 2014, darrera versió rebuda el 7 de novembre de 2014 . 